Mini Review

\title{
Childhood sensorineural hearing impairment: unsolved diagnostic issues
}

\begin{abstract}
With the successful implementation of the "Newborn Hearing Screening" program in many countries interest has been directed towards the etiologic search of childhood hearing impairment. Studies dealing with the diagnostic approach show substantial heterogeneity of the data complicating any formulation of guidelines. There is a need for a consensual, international approach in order to improve the diagnostic rate and reporting system. The various issues that arise when dealing with the etiologic evaluation of childhood hearing impairment are outlined in this opinion and actions for improvement proposed.
\end{abstract}

Keywords: hearing loss, children, diagnostics
Volume 8 Issue I - 2017

\author{
Nicolas Gurtler ${ }^{1,2}$ \\ 'Hals-Nasen-Ohren-Universitätsklinik, University Hospital Basel, \\ Switzerland \\ ${ }^{2}$ Hals-Nasen-Ohren-Klinik, Universitätskinderspital beider Basel, \\ Switzerland
}

Correspondence: N Gurtler MD, HNO-Universitätsklinik, Universitatsspital, 405I Basel, Switzerland,Tel +4 I-6I-2652525, Email Nicolas.guertler@usb.ch

Received: May II, 2017 | Published: July 12, 2017

\section{Abbreviations: HI, hearing impairment}

\section{Introduction}

Hearing impairment (HI) is the most common sensory deficit in humans with a prevalence rate of approximately 1-3 cases per 1000 of congenital hearing loss in newborns and a similar frequency for the late-onset form occurring during childhood. ${ }^{1}$ In view of a sensitive period for the development of the auditory system and therefore the need for timely hearing rehabilitation, the "Newborn Hearing Screening" program has been successfully implemented in many countries leading to earlier detection of congenital $\mathrm{HI}$ and corrective measures. ${ }^{2}$ Focus has meanwhile been redirected to the etiologic search of HI during the last years. Many benefits are associated with defining the etiology: avoidance of unnecessary examinations, improvement of prognostic counseling, reduction in health-care costs etc. Although various guidelines have been published - the latest by the British Association of Audiovestibular Physicians-,the proposed diagnostic approach for children with HI shows substantial variability across the report. ${ }^{3}$ The purpose of this opinion is to highlight the various issues associated with diagnostics of childhood $\mathrm{HI}$ and propose actions for improvement.

\section{Conclusion}

Valid evidence-based conclusions can only be made based on proper data. Instead, results are often arbitrarily reported throughout the literature dealing with the diagnostic approach of children with $\mathrm{HHI}$, thereby rendering comparison of data and pooling for analysis difficult. A recent review has outlined the extreme heterogeneity seen in the studies and emphasized the challenging interpretation of results. ${ }^{4}$ A number of issues will be listed, which should be taken into account for proper evaluation and reporting.

Complete and precise description of HI (type, onset of HI, age at diagnosis, progression, uni-/bilateral) is mandatory for reporting purposes and guiding the evaluation process. A comprehensive history follows, preferably based on a list such as established by the Joint Committee on Infant Hearing loss. ${ }^{5}$ History supports among others finding genetic causes. However, other indicators for HI might be more difficult to assess. For instance, the ototoxic level of hyperbilirubinemia has not been defined. The lack of defined positivity criteria or clear disease-cause relationships constitutes a complex and difficult issue. Stringent criteria regarding probabilities of a diseasecausing effect should be applied while interpreting results, such as genetics and serology.

Some examinations show additional benefits besides detecting the etiology, a fact supporting the decision to include them in the assessment. The prevalence of ophthalmic disorders for instance is very high between $40 \%-60 \%$, although similarly to other examinations the evidence is of low quality. ${ }^{6}$

The order and timing of examinations are especially important, as children (and often parents!) constitute a vulnerable group. Radiologic examination in small children often needs some form of anesthesia, which should be used to concurrently plan additional evaluations. However, the local setting might prevent any endeavour to optimize the organization of assessments. ${ }^{4,7}$

New technology, such as Next-Generation-Sequencing- a molecular-genetic method-,offers the most promising development in the future. Although higher genomic analytic power increases difficulties in interpreting results, clear advantages can be discerned. The high heterogeneity seen in genetic HI can be increasingly overcome by this technique with current diagnostic rates around $40 \%$ and very high sensitivity and specificity. ${ }^{8,9}$ Syndromic forms of $\mathrm{HI}$ will be detected at an early stage improving management of the patients. However, costs and the necessary availability of knowledge in bioinformatics pose an obstacle for world-wide application. Still, feasibility of this technique in smaller laboratories has been proven, which will lead to more wide-spread use. ${ }^{10}$ Incidental genomic findings while using massively-parallel-sequencing technology represent another issue, eventually raising patient's anxiety and increasing diagnostic procedures and costs.

Lastly, financial issues increasingly play a role in the discussion of any evaluation process. Although genetic testing seems to provide a cost-effective approach, complete cost-benefit analysis for examinations performed for $\mathrm{HI}$ is far from comprehensive. ${ }^{4,11}$

It is clear, that any proposal should also take into account additional restrictions such as availability of resources and incomplete collection of data. Heterogeneous practices will still continue, but a common basis should be sought. 
It is hoped, that this opinion raises the awareness of the problems seen in the etiologic evaluation of children with HHI and contributes to finding an agreement for an international standardized approach.

\section{Acknowledgements}

None.

\section{Conflicts of interest}

None declared.

\section{Funding}

None.

\section{References}

1. Gurtler N, Lalwani AK. Etiology of syndromic and nonsyndromic sensorineural hearing loss. Otolaryngol Clin North Am. 2002;35(4):891-908.

2. Sharma A, Dorman MF, Spahr AJ. A sensitive period for the development of the central auditory system in children with cochlear implants: implications for age of implantation. Ear Hear. 2002;23(6):532-539.

3. Physicians BAOA. Guidelines for aetiological investigation into severe to profound bilateral permanent childhood hearing impairment. 2015.

4. Gurtler N, Gysin C, Schmid N, et al. Bilateral congenital deafness: What investigations should be performed? Swiss Med Wkly. 2017;147:w14416.
5. American Academy of Pediatrics, Joint Committee on Infant Hearing. Year 2007 position statement: Principles and guidelines for early hearing detection and intervention programs. Pediatrics. 2007;120(4):898-921.

6. Nikolopoulos TP, Lioumi D, Stamataki S, et al. Evidence-based overview of ophthalmic disorders in deaf children: a literature update. Otol Neurotol . 2006;27(2 Suppl 1):S1-S24.

7. Yoong S, Spencer N. Audit of local performance compared with standards recommended by the national guidelines for aetiologic investigation of permanent childhood hearing impairment. Child Care Health Dev. 2005;31(6):649-657.

8. Nishio SY, Usami S. Deafness gene variations in a 1120 nonsyndromic hearing loss cohort: molecular epidemiology and deafness mutation spectrum of patients in Japan. Ann Otol Rhinol Laryngol. 2015;124(Suppl 1):49S-60S

9. Shearer AE, Smith RJ. Massively Parallel Sequencing for Genetic Diagnosis of Hearing Loss: The New Standard of Care. Otolaryngol Head Neck Surg. 2015;153(2):175-182.

10. Gurtler N, Rothlisberger B, Ludin K, et al. The Application of NextGeneration Sequencing for Mutation Detection in Autosomal-Dominant Hereditary Hearing Impairment. Otol Neurotol. 2017;38(6):900-903.

11. Preciado DA, Lim LH, Cohen AP, , et al. A diagnostic paradigm for childhood idiopathic sensorineural hearing loss. Otolaryngol Head Neck Surg. 2004;131(6):804-809. 